\title{
Software Design of an AGI System Based on Perception Loop
}

\author{
Antonio Chella \\ Dip. di Ingegneria Informatica \\ University of Palermo \\ chella@dinfo.unipa.it
}

\author{
Massimo Cossentino \\ ICAR-CNR \\ Consiglio Nazionale delle Ricerche \\ cossentino@pa.icar.cnr.it
}

\author{
Valeria Seidita \\ Dip. di Ingegneria Informatica \\ University of Palermo \\ seidita@dinfo.unipa.it
}

\begin{abstract}
According to the externalist approach, subjective experience hypothesizes a processual unity between the activity in the brain and the perceived event in the external world. A perception loop therefore occurs among the brain's activities and the external world. In our work the metaphor of test is employed to create a software design methodology for implementing an AGI system endowed with the perception loop. Preliminary experiments with a NAO humanoid robots are reported.
\end{abstract}

\section{Position Statement}

Machine consciousness concerns the attempt to model and implement in a robot those aspects of human cognition which are identified with the controversial phenomenon of consciousness, see (CM09) for a review. It does not necessarily try to reproduce human consciousness as such, insofar as human consciousness could be unique due to a complex series of cultural, social, and biological conditions. However, many authors suggested one or more specific aspects and functions of consciousness that could, at least in principle, be replicated in a robot.

It should be remembered that at the beginning of the information era there was no clear cut separation between intelligence and consciousness. Both were considered vaguely overlapping terms referring to what the mind was capable of. There was no clear boundary between intelligence and consciousness.

Today, machine consciousness assumes that there is some aspect of the mind that has not yet been adequately addressed. Therefore, scholars in the field suspect that there could be something more going on in the mind than what is currently under scrutiny in field such as artificial intelligence, cognitive science, and computer science. AGI researchers would agree that there is still a lot of work to do: better algorithms, more data, more complex, and faster learning structures. However it could be doubted whether these improvements in AGI would ever lead to an artificial agent equivalent to a biological mind or it would rather miss some necessary aspect. We, among others, suspect that classic AI missed something important; put it more clearly, we claim that facing consciousness, and in particular the subjective experience, is an essential requirement for an AGI based artifact, see, e.g. (Goe06).

In this paper we move from the externalist oriented point of view (Man06), according to which subjective experience supposes a processual unity between the activity in the brain and the perceived event in the external world. According to externalism, the separation between subject and object must be reconsidered so that the two, while maintaining their identities as different perspectives on a process, actually occur as a unity during perception.

Starting from these considerations, we developed robots aiming to exploit sensorimotor contingencies and externalist inspired frameworks (Che07). An interesting architectural feature is the implementation of a generalized perception loop based on the perceptual space as a whole. In other words, in classic feedback only a few parameters are used to control robot behavior (position, speed, etc.). The idea behind our robots is to match a global prediction of the future perceptual state (for instance by a rendering of the visual image) with the incoming data. The goal is thus to achieve a tight coupling between robot and environment. According to these model and implementations, the physical correlate of robot subjective experience would not lie in the images internally generated but rather in the causal processes engaged between the robot and the environment.

We developed a software design methodology for implementing the generalized perception loop in an AGI system. The PASSI agent oriented methodology (Cos05) has been taken into account as the starting point (CCS09) for this new design process. Until now PASSI has been used, and proved to be useful in that, for engineering robotic applications. In this work the metaphor of test is employed thus providing means for designing the elements of the perception loop. We reused, modified and integrated two process fragments coming from the Unfied Process (UP) Test Plan and Design and Test Execution, the former's aim is to identify the system functionalities to be tested, the available system resources and the test objective. The latter aims at executing test in order to identify defects and analyze 
the results.

The new PASSI allows us to design a system that, once detected the differences between expected and real behaviors (for instance during the execution of a mission), is able to autonomously tune its parameters and learn for later generalizing the knowledge to novel situations.

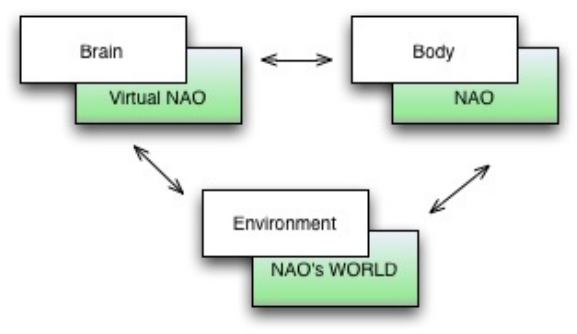

Figure 1: Realizing the Loop Brain-Body-Environment
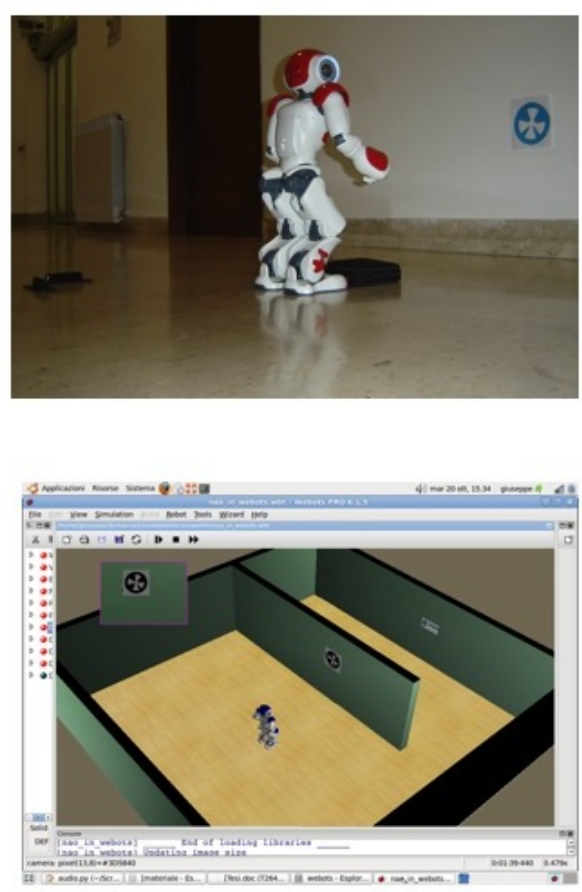

Figure 2: NAO and Virtual NAO Performing the Mission

The experiments we made aim at verifying the usability of the perception loop and the proposed software design methodology supporting that. Our aim is to create a software system able to control a robot by means of perception loops.

The robot is not equipped with "pre-compiled" preplanning abilities: we want to study the situation (and to design that) in which the robot does not know what to do in a given case, and so it queries his memory in search of a ready solution or it tries to find a novel solution exploiting his knowledge about itself, its capabilities and the surrounding world.

In particular, we experimented a humanoid robot NAO, developed by Aldebaran Robotics ${ }^{1}$, endowed with a set of primitive behaviours. In order to implement the anticipation step of the NAO perception loop, we adopted the $3 \mathrm{D}$ robot simulator Webots from $\mathrm{Cy}-$ berbotics $^{2}$. By means of Webots, we may edit NAO's movements and behaviours as well as its surrounding environment.

Figures 1 and 2 show the design and implementation of the perception loop in NAO. We exploited the fact that we use NAO and at the same time the NAO simulator, so the perception loop among brain, body ad environment (Roc05) corresponds to the loop among NAO (the real robot), the virtual NAO (the robot simulator) and the NAO's world.

It is worth noting that the use we made of the simulation is quite different from the common one: in fact, it is not used for investigating and anticipating the robot behaviour in specific working condition, but instead for producing, starting from the designed behaviours, the expected results of a mission. The simulator and NAO work separately, only when a stop condition is identified the simulation results are compared with the real NAO parameters.

Acknowledgements This work has been partially supported by the EU project FP7-Humanobs.

\section{References}

A. Chella, M. Cossentino, and V. Seidita. Towards a Methodology for Designing Artificial Conscious Robotic System. In A. Samsonovich, editor, Proc. of AAAI Fall Symposium on Biologically Inspired Cognitive Architectures BICA '09, Menlo Park, CA., 2009. AAAI Press.

A. Chella. Towards robot conscious perception. In A. Chella and R. Manzotti, editors, Artificial Consciousness. Imprinting Academic, Exter, UK, 2007.

A. Chella and R. Manzotti. Machine consciousness: A manifesto for robotics. International Journal of $\mathrm{Ma}$ chine Consciousness, 1(1):33 - 51, 2009.

M. Cossentino. From requirements to code with the PASSI methodology. In Agent Oriented Methodologies, chapter IV, pages 79-106. Idea Group Publishing, Hershey, PA, USA, June 2005.

B. Goertzel. The Hidden Pattern. BrownWalker Press, Boca Raton, 2006.

R. Manzotti. A process oriented view of conscious perception. Journal of Consciousness Studies, 13(6):7 $-41,2006$.

W.T. Rockwell. Neither brain nor ghost. MIT Press, 2005 .

\footnotetext{
${ }^{1}$ http://www.aldebaran-robotics.com

${ }^{2}$ http://www.cyberbotics.com
} 